\title{
Eigenvalue tunnelling and decay of quenched random network
}

\author{
V. Avetisov ${ }^{1,8}$, M. Hovhannisyan ${ }^{2}$, A. Gorsky ${ }^{3,4}$, S. Nechaev ${ }^{5,6}$, M. Tamm ${ }^{7,8}$, O. Valba ${ }^{8}$ \\ ${ }^{1}$ N.N. Semenov Institute of Chemical Physics of the Russian Academy of Sciences, 119991, Moscow, Russia \\ ${ }^{2}$ Chair of Programming and Information Technologies, Yerevan State University, Yerevan, Armenia \\ ${ }^{3}$ Institute of Information Transmission Problems of the Russian Academy of Sciences, Moscow, Russia, \\ ${ }^{4}$ Moscow Institute of Physics and Technology, Dolgoprudny 141700, Russia \\ ${ }^{5}$ Poncelet Laboratory, CNRS (UMI2615), Independent University of Moscow, Moscow, Russia \\ ${ }^{6}$ P.N. Lebedev Physical Institute of the Russian Academy of Sciences, 119991, Moscow, Russia \\ ${ }^{7}$ Physics Department, Moscow State University, 119992, Moscow, Russia, \\ ${ }^{8}$ Department of Applied Mathematics, National Research University Higher School of Economics, 101000, Moscow, Russia.
}

\begin{abstract}
We consider the canonical ensemble of $N$-vertex Erdös-Rényi (ER) random topological graphs with quenched vertex degree, and with fugacity $\mu$ for each closed triple of bonds. We claim complete defragmentation of large- $N$ graphs into the collection of $\left[p^{-1}\right]$ almost full subgraphs (cliques) above critical fugacity, $\mu_{c}$, where $p$ is the ER bond formation probability. Evolution of the spectral density, $\rho(\lambda)$, of the adjacency matrix with increasing $\mu$ leads to the formation of two-zonal support for $\mu>\mu_{c}$. Eigenvalue tunnelling from one (central) zone to the other means formation of a new clique in the defragmentation process. The adjacency matrix of the ground state of a network has the block-diagonal form where number of vertices in blocks fluctuate around the mean value $N p$. The spectral density of the whole network in this regime has triangular shape. We interpret the phenomena from the viewpoint of the conventional random matrix model and speculate about possible physical applications.
\end{abstract}

Investigation of critical and collective effects in graphs and networks has becoming a new rapidly developing interdisciplinary area, with diverse applications and variety of questions to be asked, see [1] for review. Ensembles of random Erdős-Rényi topological graphs (networks) provide an efficient laboratory for testing collective phenomena in statistical physics of complex systems, being also tightly linked to conventional random matrix theory. Besides investigating typical statistical properties of networks, like vertex degree distribution, clustering coefficients, "small world" structure etc, last two decades have been marked by rapidly growing interest in more refined graph characteristics, such as distribution of small subgraphs involving triads of vertices.

Triadic interactions, being the simplest interactions beyond the free-field theory, play crucial role in the network statistics. Presence of such interactions is responsible for emergence of phase transitions in complex distributed systems. First example of a phase transition in random networks, known as Strauss clustering 2, has been treated by the Random Matrix Theory (RMT) in 3. It was argued that, when the increasing fugacity, $\mu$, the system develops two phases with essentially different triad concentrations. At large $\mu$ the system falls into the Strauss phase with the single clique of nodes. The condensation of triads is a non-perturbative phenomenon identified in 4 with the 1st order phase transition in the framework of mean-field cavity-like approach.

Similar critical behavior was found in [5] for the vertexdegree-conserved ER graphs. It was demonstrated in the framework of the mean-field approach that the phase transition takes place in this case as well. The hysteresis for dependence of the triad concentration on the fugacity, $\mu$ also has been observed in [5]. For bi-color networks with conserved vertex degree a new phenomena of a wide plateau formation in concentration of black-white bonds as a function of the fugacity of unicolor triples of bonds has been found in [6].

Note that all these models are essentially athermic: in the absence of an external field, the partition function of the network is a purely combinatorial object with no interactions and no temperature dependence, and its evolution can be regarded as a Langevin dynamics in the stochastic quantization framework. The solution to the corresponding Fokker-Planck equation at the infinite stochastic time yields the exact quantum ground state of the model.

Here we provide deeper insight into the structure of phase transition in non-directed vertex-degree-conserved Erdős-Rényi random graphs, with the number of fully connected triples of vertices, $n_{\triangle}$, controlled by $\mu$. The numerical simulations possess a number of striking phenomena: (i) above some critical value, $\mu_{c}$, the network splits into the maximally possible number of clusters, identified as cliques, (ii) the number of cliques for large graphs is fixed exclusively by the average vertex degree at the network preparation, $p$, (iii) above $\mu_{c}$ the spectral density of the whole network has the triangular shape, typically observed for scale-free networks. This behavior differs significantly from the unconstrained Strauss model, where above the transition point the single maximally connected clique is formed.

Qualitatively the formation of the Strauss condensate in the unconstrained network can be understood as follows. For $\mu=0$ the system lives in the largest entropic basin corresponding to some equilibrium distribution of triads. As $\mu$ is increasing, the triad distribution gets gradually more skewed. In the limit $\mu \rightarrow \infty$, the entropic effects become irrelevant, and the network approaches 
the state with the largest energy, $\mu n_{\triangle}$. Depending on the shape of the entropy, the dependence $n_{\triangle}(\mu)$ can be either a smooth function, or can undergo abrupt jumps at some particular values of $\mu_{c}$ typical for first-order phase transitions. In contrast, in the vertex-degree-conserved model the constraints prohibit the formation of a large single clique since the constraints prevent of complete mixing. Therefore the system does its best and splits into the maximally possible number of allowed cliques, which is the true ground state for the network with quenched vertex degree. The same decay occurs for so-called "regular random networks" (which have the one and the same degree $N p$ in all nodes).

Any topological graph or collection of graphs can be encoded by the adjacency matrix, $A$ [7]. In the quenched model, within the transition region, isolated eigenvalues of $A$ form the second zone in the spectrum and correspond one-by-one to clusters in the large network (see [810] for general description). Above the transition point, the spectral density (SD), $\rho(\lambda)$, of the adjacency matrix of each clique (almost fully connected subgraph) is the same as the spectral density of the sparse matrix, and has Lifshitz tails typical for 1D Anderson localization, as discussed in [11]. The spectral density of the whole network has a triangle-like shape typically seen in scale-free networks. Note, however that in our system the set of vertex degrees is quenched at the preparation, and has the Poisson distribution.

Found behavior has many parallels in the random matrix model. The effective potential of the Strauss model involves the quadratic and cubic terms [3]. The conventional random matrix model attributes the particular Riemann surface to the effective potential due to the loop equations. In the cubic case it yields the genus one Riemann surface for the SD, therefore emergence of two zones in the support of $\rho(\lambda)$ of the adjacency matrix, is natural. The distribution of eigenvalues between two zones, in the random matrix model corresponds to the symmetry breaking $U(N) \rightarrow U(N-k) \times U(k)$, where $k$ is the number of eigenvalues in the second zone, and is essentially nonperturbative. In the network case the symmetry breaking pattern could be more subtle since we do not know explicitly the measure in the ensemble of adjacency matrices. The eigenvalue tunnelling between two zones is fairly general phenomena in the matrix model framework (see [17] for the review) meaning the formation of a kind of extended coherent object, like a baby Universe. In our case, such tunnelling leads to the dense droplets (cliques) formation, being the peculiar example of the global symmetry breaking.

The model under consideration is essentially the same as in [5]. We consider the canonical ensemble of topological Erdős-Rényi graphs with quenched vertex degree. Each closed triple of bonds (the closed triadic motif) is weighted with $\mu$. The partition function of the system is

$$
Z(\mu)=\sum_{\{\text {states }\}}^{\prime} e^{-\mu n_{\Delta}}
$$

where the prime in (1) means that the summation runs over all possible configurations of nodes, under the condition of fixed degree $v_{i}$ in each vertex $i(i=1, \ldots, N)$ of the graph. The direct computation of $Z(\mu)$ in numerical simulations is very cumbersome, so, usually the numeric algorithm runs as follows. The initial realization of ER network is prepared by connecting any randomly taken pair of vertices with the probability $p$. Then, one randomly chooses two arbitrary links, say, between vertices $i$ and $j,(i j)$ and between $k$ and $m,(k m)$, and reconnect them, getting new links $(i k)$ and $(j m)$. Such reconnection conserves the vertex degree [18]. Now one applies the standard Metropolis algorithm with the following rules: i) if under the reconnection the number of close triples is increased, a move is accepted, ii) if the number of close triples is decreased by $\Delta n_{\triangle}$, or remains unchanged, a move is accepted with the probability $e^{-\mu \Delta n_{\Delta}}$. Then the Metropolis algorithm runs repeatedly for large set of randomly chosen pairs of links, until it converges. In [5] only the behavior of $\left\langle n_{\triangle}(\mu)\right\rangle$ was considered. Here we turn to more detailed investigation of the ground state in the constrained network.

We state that, given the probability, $p$, of the bond formation in the initial network, the evolving network splits into the maximal number of clusters, $\left[p^{-1}\right]$ (where [...] means the integer part), being independent on the particular set of corresponding vertex degrees, $\left\{v_{1}, \ldots, v_{N}\right\}-$ see the Fig.1. In other words, the system with the maximal number of clusters (cliques) is the proper ground state of the model. Thus, the system falls into the new phase different from the Strauss one. Below we confirm this by analyzing the spectral density of the adjacency matrix.

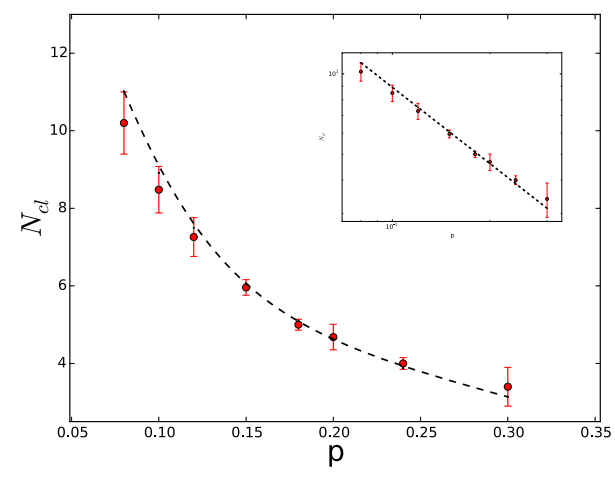

FIG. 1: The number of clusters $N_{c l}$ as a function of the probability $p$ in ER graph. The numerical data are obtained by averaging over 100 randomly generated graphs up to 512 vertices. Numerical values are fitted by the curve $p^{-0.95}$; the behavior in doubly logarithmic scale is shown in the insert.

For any particular quenched network pattern and $\mu<\mu_{c}$, the spectral density has the shape typical for 
ER graphs with moderate connection probability, $p=$ $O(1)<1$, being the Wigner semicircle with a single isolated eigenvalue apart. At $\mu_{c}$ the eigenvalues decouple from the main core and a collection of isolated eigenvalues forms the second zone. The number of isolated eigenvalues exactly coincides with the number of clusters formed above $\mu_{c}$. This perfectly fits with the result of [9]. Averaging over ensemble of graphs patterns smears the distribution of isolated eigenvalues in the second zone. Above $\mu_{c}$ the support of SD in the first (central) zone shrinks and the second zone becomes dense and connected - see the Fig, 2

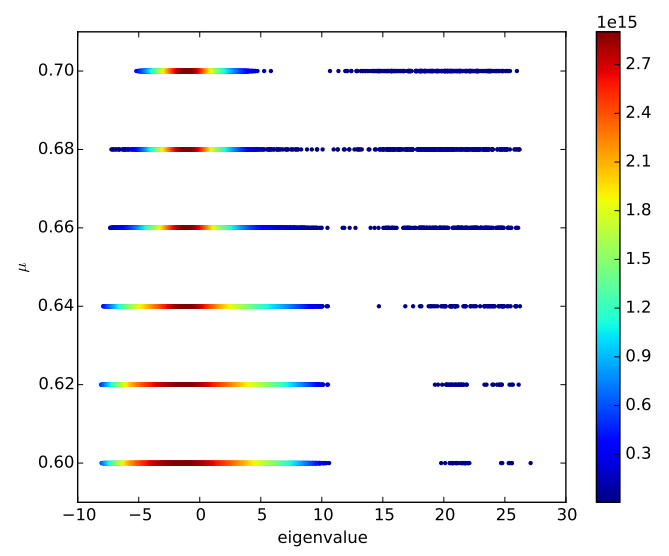

FIG. 2: Spectral density of ensembles of ER graphs for different $\mu$. The numerical results are obtained for $50 \mathrm{ER}$ graphs of 256 vertices and $p=0.08$.

We have investigated SD inside each cluster $(\mathrm{Cl})$ aiming to prove that $\mathrm{Cl}$ is almost full graph (clique). We see that the shape of SD is drastically changed at $\mu_{c}$, see the Fig 3, were SD in the first zone below and above $\mu_{c}$ are shown. The triangle-shaped SD is typical for the scalefree networks [12, 13, however in our case the vertex degree is fixed at network preparation and can be only redistributed between cliques. The remarkable point is that the SD evaluated for each particular clique exhibits a hierarchical set of resonance peaks typical for sparse matrices, which have been analytically investigated in [1].

Using duality between the spectrum of sparse and almost full graphs [14, we see from the Fig, 4 that SD in almost complete graph fits perfectly the shifted SD of sparse matrix ensemble, meaning that our identification of clusters with cliques and separated eigenvalues is true. The striking difference between the SD of single clique and the whole network indicates that the triangle-shape $\mathrm{SD}$ of the whole networks occurs due to the inter-clique connections.

It is very instructive to compare the typical adjacency matrices in the ground state of the ER network with and without constraints at the same value of $p$. The corresponding matrices are presented at Fig.5, where different

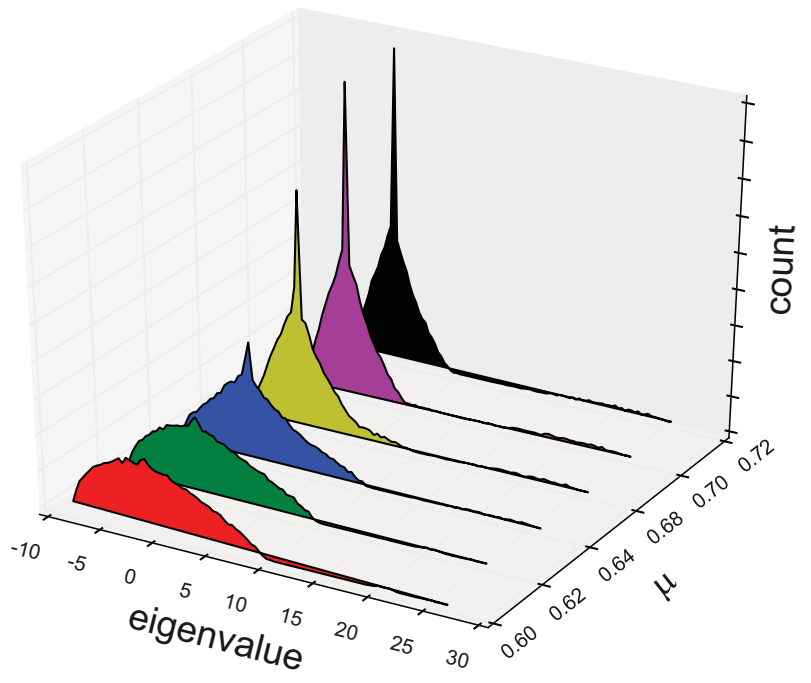

FIG. 3: The spectral density in the ensembles of ER graphs for different $\mu$. The numerical results are obtained for the ensembles of 50 random ER graphs of 256 vertices and the probability $p=0.08$.
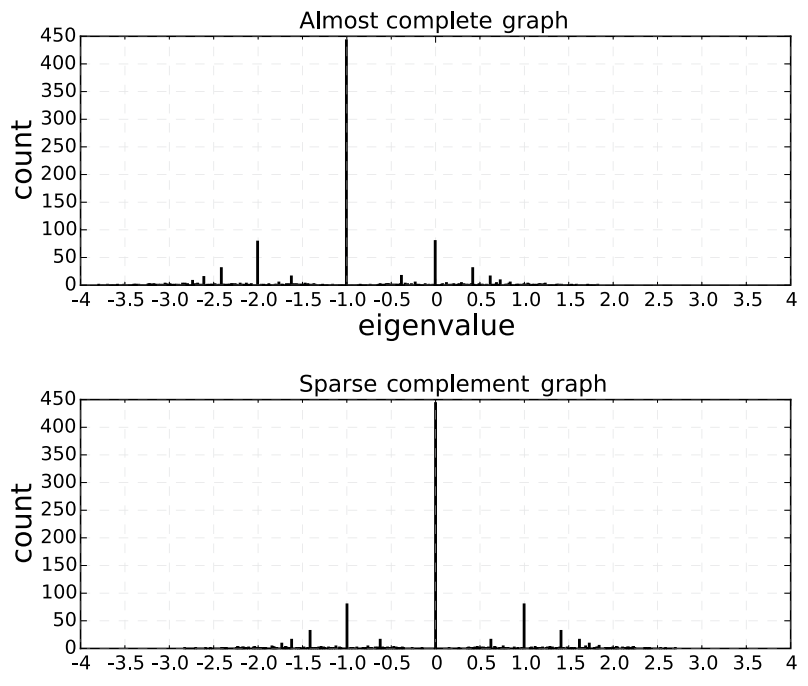

FIG. 4: The duality between spectral densities of ensembles of almost fully connected graphs and their sparse complements.

phases of the ground states are clearly seen. The ground state in the Strauss phase consists of a single complete graph corresponding to the block in adjacency matrix of some size, $k$, while the ground state of the quenched network involves $\left[p^{-1}\right]$ almost complete graphs corresponding to blocks (cliques) in the adjacency matrix with fluctuating sizes $N_{i}\left(\sum_{i} N_{i}=N\right)$ and the mean value in the clique, $N_{c l}=\left\langle N_{i}\right\rangle=N /\left[p^{-1}\right] \approx N p$.

To visualize the kinetics, we proceed as follows. First, we enumerate vertices at the preparation condition in arbitrary order and run the Metropolis stochastic dynamics. When the system is equilibrated and the cliques are formed, we re-enumerate vertices sequentially according to their belongings to cliques. Then we restore corre- 


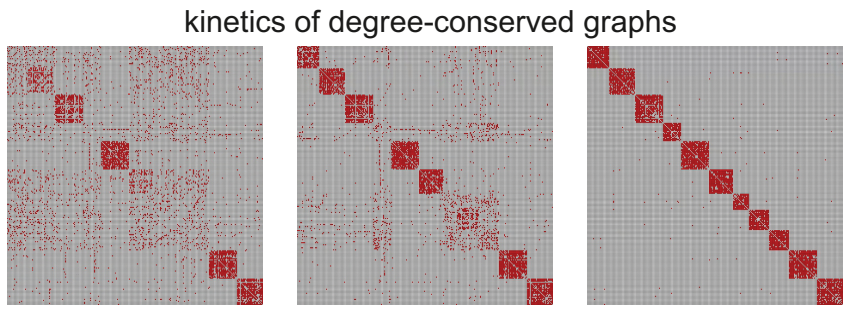

kinetics of non-conserved graphs

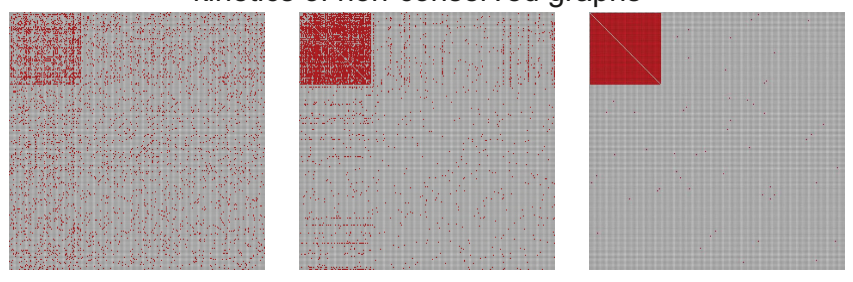

FIG. 5: Few typical samples of intermediate stages of the network evolution: upper panel - evolution with fixed vertex degree; lower panel - eolution with non-fixed vertex degree.

sponding dynamic pathways back to initial configuration.

In the sparse regime there is the percolation phase transition in the ER network at $p_{\text {perc }}=N^{-1}$ corresponding to the critical behavior of the $q=1$ Potts model. Due to the duality, one could expect the dual percolation phase transition in the clique happens at $\bar{p}_{\text {perc }}=1-N_{c l}^{-1}$. The dual percolation corresponds to the creation of dislocations in the cliques extended through the whole droplet. The right tail of the spectral density in the first zone behaves as

$$
\left.\rho(\lambda)\right|_{\lambda \rightarrow \lambda_{\max }} \sim e^{-c / \sqrt{\lambda_{\max }-\lambda}}
$$

( $c$ is some positive constant) and is, as shown in [1] for sparse ensembles, a manifestation of a Lifshitz tail for the Andersen localization.

Let us interpret our results in terms of the random matrix models postponing the detailed analysis for the separate publication. The unconstrained Strauss model was analyzed in this context in [3. The choice of the classical potential in the large- $N$ network matrix model is straightforward: the quadratic term fixes the number of links in the network, while the cubic term encounters triangles since $\operatorname{Tr} M^{3}=N_{\text {triangles }}$. Therefore the matrix model for the Strauss model reads as [3]

$$
Z\left(t_{k}\right)=e^{F}=\int d M e^{g^{-1} V(M)},
$$

where $V(M)=a \operatorname{Tr} M^{2}+\mu \operatorname{Tr} M^{3}$ and the parameter $a=\log \left(p^{-1}-1\right)$ for unconstrained ER network. The Strauss model exhibits the phase transition at the critical value of the chemical potential for triangles and is exact counterpart of the phase transition known in the matrix model description of the pure $2 \mathrm{~d}$ quantum gravity [15]. However one should take care comparing the random matrix models and the random adjacency matrix since the measures in the matrix integrals are different.
There is Riemann surface associated with any matrix integral where spectral density in defined on

$$
y^{2}=\left(V^{\prime}(x)\right)^{2}+f(x)
$$

where $y$ and $x$ are complex variables. The function $y(x)$ is related to the matrix model resolvent, $\omega(x)=\left\langle\operatorname{Tr} \frac{1}{M-x}\right\rangle$, as follows

$$
y(x)=2 \omega(x)+V^{\prime}(x)
$$

For the potential $V=a x^{2}+\mu x^{3}$ the Riemann surface has genus one. The coefficients of polynomial $f(x)$ are fixed by the filling fractions, $N_{i}$, around extrema of the potential $V(x)\left(V_{e f f}^{\prime}\left(a_{i}\right)=0\right)$. In the cubic case for the Strauss model the eigenvalues are distributed in two zones and if $k$ eigenvalues are in the second zone, the adjacency matrix of the ground state involves the size $k$ block filled by ones.

Thus, the second zone always exists, however in the unconstrained case all, but one, eigenvalues belong to the first (central) zone with the Wigner semicircle distribution. The situation in the constraint-driven case is different since fixed number of eigenvalues pass from the first zone to the second one through the gap. From the conventional matrix model viewpoint the question can be formulated a follows: why the fixed number of eigenvalues are forced to tunnel between zones above the critical value of $\mu$ in the constrained model? The eigenvalue tunnelling is very general phenomena, first discussed in the context of 2D gravity in [16. The review of the general place of the eigenvalue tunnelling in topological strings and the matrix models can be found in [17. Depending of the physics described by the matrix model it corresponds to the account of FZZT branes in the noncritical string, baby Universe creation in the quantum gravity, or the creation of D-brane domain wall in the supersymmetric gauge theory.

It is convenient to introduce the constraints into the matrix model via Lagrangian multiplies. They yield the linear term in the action $\operatorname{Tr} \Lambda X$ with $\Lambda_{i j}=$ $\left\{z_{1} \delta_{1 j}, \ldots, z_{N} \delta_{N j}\right\}$ and one has to integrate over the Lagrangian multipliers, $z_{i}$. That is we have a very peculiar version of the two-matrix model. The situation resembles the symmetry breaking by the Wilson loop observables in the matrix model framework. In our case, qualitatively the form of the constraint "selects the representation" of the Wilson loop and fixes the symmetry breaking pattern. We can easily recognize that the pattern of the symmetry breaking realized in numerical simulations indeed, is consistent with the form of constraint. Therefore, qualitatively we see that the matrix model is consistent with two results of the numerical simulations: presence of multi-zonal support for the spectral density, and the formation of cliques, by the mechanism of inter-zone eigenvalue tunnelling.

The transition from the Wigner semicircle to the triangle-like SD is known in the matrix model description of the Dirac operator spectrum in QCD [19, 20] 
and admits deep physics behind. If we scan QCD at finite volume at energies, smaller then the Thouless energy, $E_{T}$, which divides the "ergodic" and "diffusive" regions in generic systems, the Dirac operator considered as the Hamiltonian in $4+1$ space-time enjoys the semicircle spectrum. In this regime the spectrum is evaluated via the instanton liquid chiral matrix model and is saturated by the constant modes. On the other hand at $E \gg E_{T}$, the non-constant modes are important and the $\mathrm{SD}$ at small $\lambda$ reads as

$$
\rho(\lambda)=\rho(0)-c|\lambda|
$$

where $c>0$ is defined by the exchange of two soft Goldstone modes between two coherent states with scalar quantum numbers 21. Such soft modes are present due to the spontaneous chiral symmetry breaking. This perfectly fits with our observation that the triangle-shape $\mathrm{SD}$ in the multi-clique phase definitely is due to the links between pairs of cliques. The global symmetry is broken in our model above the phase transition as well, hence we could expect the presence of soft modes which would play the role of diffusons and could provide the required inter-clique interaction.

It is worth mention that nowadays the large- $N$ matrix model is interpreted as the theory of the open string tachyon on the $N$ unstable D0 or ZZ branes [22, 23]. The final state of the evolution of the unstable system is the coherent state of the closed string modes or stable D branes. We have clear counterpart of this phenomena in our network as formation of the highly coherent states set of cliques. Its number is fixed by the model from the very beginning. The system on the stable FZZT branes identified as the Kontsevich-like matrix model [24, 25] seems to be relevant for the description of the multiclique phase of our model.

In this Letter we described a decay of the constrained topological network into multi-clique phase above some critical value of the chemical potential for triads. The decay has been analyzed via evolution of the spectral density of the adjacency matrix. The ground state of the system above the transition point is identified as interacting multi-clique states. The eigenvalue tunnelling is the key point in our problem. This issue is a general nonperturbative phenomenon in matrix models describing formation of extended objects, and our finding could provide new insight on it. We believe that our model sheds additional light on the formation of stable D-brane from unstable ones connected by strings. The similar spectral analysis of the constrained multicolor network will be presented in a forthcoming publication [26].

The imposed constraint is not exotic, being typical for the chemical, biological and social networks. The phenomena we found can be considered as the operational tool to split the network into the optimal droplets of almost full subgraphs (cliques) for generic random networks. Varying the constraints, the required design of the ground state in the quantum network with the proper symmetry breaking pattern can be manufactured.

The important finding concerns the emergence of the second zone and the genus one Riemann surface. It immediately suggests that we could search for the action of the modular group on the torus modulus. Such spectral Riemann surface nowadays is familiar in many examples and usually the filling of the A-cycle of the torus corresponds to the "perturbative" degrees of freedom while the filling of the B-cycle corresponds to the "nonperturbative" solitonic degrees of freedom which can be build from the large number of the perturbative ones. The monopole and gauge boson are familiar example of this phenomena. In our case we see the similar situation: the main "perturbative" zone corresponds to the small perturbations, while the second zone is filled by the nonperturbative solitonic cliques. It would be interesting to develop the "particle-soliton" duality for the network ground state from the modular properties of the spectral torus. We postpone this challenging question for the separate study.

We conclude by mentioning the possible relation of random network with quenched vertex degree with some known physical models. In the context of quantum gravity (see for review 27 29] the following question can be posed. The model under consideration is topological and does not involve the metric structure. Is it possible to interpret clustering as the appearance of the effective metric, see [30] for the recent discussion. We could conjecture that the phase transition considered corresponds to the transition from the topological $\left\langle g_{\mu \nu}\right\rangle=0$ phase to the geometrical phase $\left\langle g_{\mu \nu}\right\rangle \neq 0$ of the network. The geometric phase could be related with the polymer phase of the $2 \mathrm{~d}$ quantum gravity.

Another application deals with the budding phenomena (formation of bubble-like vesicles due to spontaneous curvature) in lipid membranes 31. If the membrane is liquid, the material can be redistributed over the whole tissue and only one vesicle is typically formed. However, in presence of quenched disorder in the membrane, the redistribution of the material over the whole sample is blocked and the formation of multi-vesicle phase seems plausible.

We are grateful to D. Kryukov and A. Mironov for the useful discussions. A.G. thanks the SCGP at Stony Brook University where the part of the paper has been done for the hospitality and support during the program "Geometry of the Quantum Hall State". The work of A.G. was supported by grant of Russian Science Foundation 14-050-00150 for IITP. 
[1] S. Dorogovtsev, A. Goltsev and J. Mendes " Critical phenomena in complex networks"' arXiv:0705.0010

[2] D. Strauss, On a General Class of Models for Interaction, SIAM Rev. 28, 513 (1986)

[3] Z. Burda, J. Jurkiewicz, and A. Krzywicki, Network transitivity and matrix models, Phys. Rev. E 69, 026106

[4] J. Park and M. E. J. Newman, Solution for the properties of a clustered network, Phys. Rev. E 72, 026136

[5] M.V. Tamm, A.B. Shkarin, V.A. Avetisov, O.V. Valba, and S.K. Nechaev, Islands of Stability in Motif Distributions of Random Networks, Phys. Rev. Lett. 113, 095701

[6] V. Avetisov, A. Gorsky, S. Nechaev and O. Valba, "Spontaneous Symmetry Breaking and Phase Coexistence in Two-Color Networks," Phys. Rev. E 93, no. 1, 012302 (2016) doi:10.1103/PhysRevE.93.012302 arXiv:1506.00205 [cond-mat.stat-mech]].

[7] S.N. Dorogovtsev, A.V. Goltsev, J.F.F. Mendes, A.N. Samukhin "'Spectra of complex networks"' Phys. Rev. E 68, 046109 (2003)

[8] Raj Rao Nadakuditi, M. E. J. Newman "Graph spectra and the detectability of community structure in networks"' Phys. Rev. Lett. 108, 188701 (2012) arXiv:1205.1813

[9] Nadakuditi, Raj Rao; Newman, M.E. J. "'Spectra of random graphs with arbitrary expected degrees"' Physical Review E, vol. 87, Issue 1, id. 012803 arXiv:1208.1275

[10] Zhang, Xiao; Nadakuditi, Raj Rao; Newman, M. E. J. "Spectra of random graphs with community structure and arbitrary degrees"' Physical Review E, Volume 89, Issue 4, id.042816 arXiv:1310.0046

[11] V. Avetisov1, P.L. Krapivsky, and S. Nechaev, Native ultrametricity of sparse random ensembles, J. Phys. A: Math. Theor. 49 (2016) 035101 (25pp) doi:10.1088/17518113/49/3/035101 arXiv:1506.05037.

[12] Illes J. Farkas, Imre Derenyi, Albert-Laszlo Barabasi, Tamas Vicsek "Spectra of "Real-World" Graphs: Beyond the Semi-Circle Law", Physical Review E 64, 026704:1-12 (2001)

[13] K.-I. Goh, B. Kahng, D. Kim " 'Spectra and eigenvectors of scale-free networks"' Phys. Rev. E 64, 051903 (2001)

[14] T. Tao and V. Vu, Random matrices: universality of local eigenvalue statistics, Acta Math., 206(1):127204 (2011);

[15] P. H. Ginsparg and G. W. Moore, "Lectures on 2-D gravity and 2-D string theory", hep-th/9304011

[16] F. David, "Nonperturbative effects in matrix models and vacua of two-dimensional gravity", Phys. Lett. B 302, 403 (1993) doi:10.1016/0370-2693(93)90417-G hepth/9212106.

[17] M. Mario, "Lectures on non-perturbative effects in large $N$ gauge theories, matrix models and strings", Fortsch. Phys. 62, 455 (2014) doi:10.1002/prop.201400005 arXiv:1206.6272 [hep-th]].
[18] S. Maslov and K. Sneppen, Specificity and Stability in Topology of Protein Networks, Science, 296, 910 (2002)

[19] J. C. Osborn, D. Toublan and J. J. M. Verbaarschot, From chiral random matrix theory to chiral perturbation theory", Nucl. Phys. B 540, 317 (1999) doi:10.1016/S0550-3213(98)00716-0 hep-th/9806110.

[20] R. A. Janik, M. A. Nowak, G. Papp and I. Zahed, "Chiral disorder in QCD," Phys. Rev. Lett. 81, 264 (1998) doi:10.1103/PhysRevLett.81.264 hep-ph/9803289.

[21] A. V. Smilga and J. Stern, "On the spectral density of Euclidean Dirac operator in QCD," Phys. Lett. B 318, 531 (1993).

[22] J. McGreevy and H. L. Verlinde, "Strings from tachyons: The c=1 matrix reloaded," JHEP 0312, 054 (2003) doi:10.1088/1126-6708/2003/12/054 hep-th/0304224.

[23] I. R. Klebanov, J. M. Maldacena and N. Seiberg, "Dbrane decay in two-dimensional string theory," JHEP 0307, 045 (2003) doi:10.1088/1126-6708/2003/07/045 hep-th/0305159.

[24] D. Gaiotto and L. Rastelli, "A Paradigm of open / closed duality: Liouville D-branes and the Kontsevich model," JHEP 0507, 053 (2005) doi:10.1088/1126$6708 / 2005 / 07 / 053$ hep-th/0312196.

[25] M. Aganagic, R. Dijkgraaf, A. Klemm, M. Marino and C. Vafa, "Topological strings and integrable hierarchies," Commun. Math. Phys. 261, 451 (2006) doi:10.1007/s00220-005-1448-9 hep-th/0312085.

[26] V. Avetisov, A. Gorsky, M. Hovhannisyan, S. Nechaev, M. Tamm and O. Valba "Critical behavior of multicolor constrained topological networks via eigenvalue tunneling"' to appear

[27] J. Ambjorn, R. Loll, Y. Watabiki, W. Westra and S. Zohren, "New aspects of two-dimensional quantum gravity" Acta Phys. Polon. B 40, 3479 (2009) arXiv:0911.4208 [hep-th]].

[28] G. Bianconi and C. Rahmede, "Network geometry with flavor: from complexity to quantum geometry," Phys. Rev. E 93, no. 3, 032315 (2016) doi:10.1103/PhysRevE.93.032315 arXiv:1511.04539 [cond-mat.stat-mech]]

[29] C. A. Trugenberger, "Quantum Gravity as an Information Network: Self-Organization of a 4D Universe", Phys. Rev. D 92, $084014 \quad$ (2015) doi:10.1103/PhysRevD.92.084014 arXiv:1501.01408 [hep-th]].

[30] D. Krioukov, "Clustering implies geometry in networks", Phys. Rev. Lett. 116, 208302 (2016) arXiv:1604.01575

[31] R. Lipowsky, Budding of membranes induced by intramembrane domains, J. de Physique II, 2, 1825 (1992); F. Julicher, R. Lipowsky, Domain-Induced Budding of Vesicles, Phys. Rev. Lett., 702964 (1993) 